\title{
Diffusion processes in the transition layer of the Earth's magnetosphere
}

\author{
A.S.Prokhorenkov ${ }^{1 *}$ L. V. Kozak ${ }^{1}$, A. T. Y.Lui ${ }^{2}$, I. V. Gala ${ }^{1}$ \\ ${ }^{1}$ Taras Shevchenko National University of Kyiv, Glushkova ave. 4, 03127, Kyiv, Ukraine \\ ${ }^{2}$ Johns Hopkins University Applied Physics Laboratory, Laurel MD, USA
}

\begin{abstract}
Turbulence has a different nature in the interplanetary magnetic field and in the transition region, thus it requires a different type of analysis. The "Cluster 2" satellite mission provides magnetic measurements with a temporal resolution of $22.5 \mathrm{~Hz}$. We analysed the evolution of the probability density function over time, as well as that of the structural function. From the analysis we can conclude that for small time scales, the fluctuation distribution differs significantly from the Gaussian. Furthermore, we see that in the foreshock region, the fluctuation becomes almost Gaussian. Using the extended self-similarity structure function we compare the experimental data with the Kolmogorov K41 model. Calculated diffusion coefficients have a good agreement with the analysis of the probability density function and this can prove the existence of superdiffusion processes in the transition region of the Earth's magnetosphere.
\end{abstract}

Key words: solar wind-magnetosphere interactions nonlinear phenomena; transport processes

\section{INTRODUCTION}

A new type of instability arises in inhomogeneous plasma, as compared with a classical description. In the case of evolution of these instabilities, the plasma can become a turbulent medium. In addition, the transverse diffusion of plasma in the magnetic layer can play a significant role in areas where magnetic reconnections are ineffective. Thus, diffusion in the absence of actual particle collisions can be ensured as a wave-particle interaction (anomalous resistive diffusion). Since gradients of density, temperature, magnetic field and flow are observed in the transition region of the magnetosphere, a large number of instabilities for anomalous diffusion evolves. A number of macro-instabilities may also contribute to the diffusion. The Kelvin-Helmholtz instability and eddy turbulence are the most important instabilities to evolve the inhomogeneous plasma characteristics. Both mechanisms can promote mixing of the plasma on a large scale, and can create the necessary conditions for micro-instabilities. Chaotic electric and magnetic fields that occur, lead to anomalous transport processes that are orders of magnitude higher than that of classical processes. Macroscopic flows of particles, momentum, and energy are defined not only by the mean field and profiles, but also by the fluctuation spectrum [17].

The notions and concepts of anomalous dynamic properties, such as long-range spatial or temporal correlations manifested in power-laws, stretched exponentials, or non-Gaussian probability distribution functions (PDFs), have been predicted and observed in numerous systems from various disciplines including physics, chemistry, engineering biology, meteorology, astrophysics, and others. The standard tools to describe anomalous dynamics are continuous time random walks $[7,8,20]$ and fractional dynamical equations $[15,16,21]$.

The anomalous features usually stretch over the entire data window, but there exist examples when they develop after an initial period of sampling (finite size/time effects), or they may be transient, i. e., eventually the nature of anomalous process turns into normal transport or relaxation dynamics. The most fundamental definition of anomaly is the deviation of the mean squared displacement $\left\langle(\Delta r)^{2}\right\rangle=$ $\left\langle(r-\langle r\rangle)^{2}\right\rangle \sim D t^{\propto}$ from the 'normal' linear dependence $\left\langle(\Delta r)^{2}\right\rangle \sim D t$ over time. Here $D$ is the generalized diffusion constant. The anomalous diffusion exponent $\alpha \neq 1$ determines whether the process will be categorized as subdiffusive (dispersive, slow, etc.) if $0<\alpha<1$, or superdiffusive (enhanced, fast) if $1<\alpha$. Usually, the domain $1<\alpha \leq 2$ is considered, $\alpha=2$ being the ballistic limit described by the wave equation, or its forward and backward modes [14]. Processes with $\alpha>2$ are known, such as the Richardson pair diffusion in fully developed turbulence [18].

Generally, one can not create a closed system of equations describing the anomalous transport processes; the results are mostly limited to semiquantitative assessments. The weak turbulence,

*andrew.prokhorenkov@gmail.com

A.S. Prokhorenkov, L. V. Kozak, A. T. Y.Lui, I. V. Gala, 2015 
with quasi-linear approximation, is a case when the anomalous transport processes can be analytically described.

Therefore, it is necessary to determine the statistical properties of the environmental parameter fluctuations from the experiment which are related to the scale invariance, and get estimates for the type of diffusion processes. This will qualitatively and quantitatively describe transport processes in different regions of the magnetosheath.

\section{METHODS TO DETERMINE THE \\ TYPE OF DIFFUSION PROCESSES}

\section{EVOLUTION OF ThE FLUCTUATIONS PDF}

Probability distribution of fluctuation amplitudes of a random process obeys the Gaussian law (the socalled normal law) $[3,10]$. The dependence of the maximum probability density function in the analysis of data series $X(t)$ at different time scales can be approximated by a power law: $P_{\tau}(0) \sim \tau^{-s}$, where $\tau$ is the scale shift over time, and $s$ is a parameter that characterizes the homogeneity or heterogeneity of the studied process (for Gaussian distribution $s \sim 0.5$ and the presence of heterogeneity $s>0.5[3,6]$.

From the behaviour of the PDF the spatial or temporal scales where the PDF losses the Gaussian properties, can be defined.

Evaluation of particle biases can be carried out using the "Levy flight" (Levy flights) [2, 22]: $\left\langle X^{2}(\tau)\right\rangle \propto \tau^{2 s}$, where $s$ is the maximum exponent of magnetic field fluctuations PDF $P_{\tau}(0)$.

\section{Statistical averages properties.}

For the turbulent field $X(t)$, the structural function (statistical average) of order $q$ is defined as a statistical average over the ensemble of relations [9]: $\delta_{\tau} X=X(t+\tau)-X(t), S_{q}(t)=\left\langle\left|\delta_{t} X\right|^{q}\right\rangle \sim t^{\zeta(q)}$, where the exponent $\zeta(q)$ - describes the type of process and turbulent diffusion properties of the medium. The linear dependence of $\zeta(q)$ indicates homogeneity of turbulent processes (particularly, for the Kolmogorov model $-\zeta(q)=q / 3$, and Iroshnikov-Kraichnan model $\zeta(q)=q / 4)[9,12,13$, $23]$. For turbulence intermittence of high order structure functions there is a nonlinear dependence of $\zeta(q)$ on $q$. This reflects the deviations from the Gaussian law [5]. In addition, the structure functions of high orders allow one to characterize the properties of heterogeneity on small scale process.

Analysis of experimental data has a great importance to the presence of extended properties of selfsimilarity (ESS), for a power law of the structure functions $S_{q} \sim S_{p}^{\zeta(q) / \zeta(p)}$ and allows one to characterize fluctuations in the turbulent flow for a large range of Reynolds numbers [1]. Using the extended self-similarity property, the good accuracy values of $\zeta(q)$ and diffusion properties of the plasma can be evaluated. To interpret the nonlinear dependence $\zeta(q)$ the log-Poisson model is used, for which $\zeta(q)$ is given by $[4,11,19]$ :

$$
\zeta(q)=(1-\Delta) \frac{q}{3}+\frac{\Delta}{1-\beta}\left[1-\beta^{\frac{q}{3}}\right],
$$

where $\beta$ and $\Delta$ are parameters that characterize the intermediate and singularity processes, respectively.

As a result of ESS-analysis for log-Poisson scaling parameters $\beta$ and $\Delta$ are used to determine the characteristics of turbulent plasma transport. The generalized diffusion coefficient depends on the $\zeta(q)$ as [22]:

$$
D \propto t^{R},
$$

where $R=\Delta(1 / \beta-l)$.

This relation is used to evaluate transport properties in a heterogeneous environment. In general, the parameter $R$ is defined by the fractal properties of the medium.

Thus, there is a relation between the exponent that characterizes the evolution of PDF, and the parameter $R$. Displacement law for the particles is given by the relation: $\left\langle\delta X^{2}(\tau)\right\rangle \propto D \tau \propto \tau^{2 s} \propto \tau^{\alpha}$, with order of $2 S=1+R[2,22]$. Where, normal diffusion corresponds to $\alpha=1$, and the convective (ballistic) motion is characterized by the $\alpha=2$.

\section{SATELLITES USED FOR MEASUREMENT}

We used the data from "Cluster 2" space mission. We analysed 5 events of satellites $\mathrm{C} 1$ passing the magnetosheath from 2004 to 2010, with a temporal resolution of $22.5 \mathrm{~Hz}$. The examples of analysed magnetic field fluctuations are shown in Fig. 1. In two events out of five (2009/05/11 and 2010/03/31), the satellite crossed the magnetosheath, moving from the magnetopause region to the interplanetary medium. In other cases it was moving from the solar plasma wind medium.

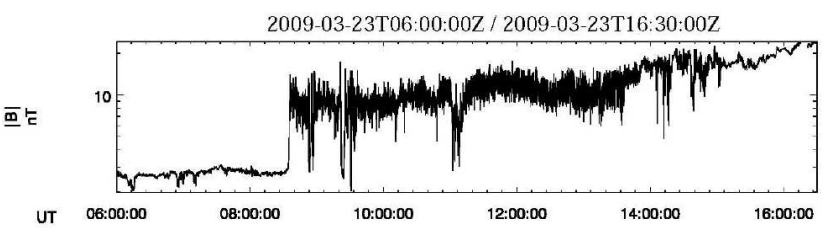

Fig. 1: Example of analysed magnetic field.

In different regions of magnetosheath there are different levels of magnetic field fluctuations observed:

- in foreshock region, the variance of variations normalized with the current mean value is $\delta B / B=0.1-0.25$;

- after crossing the shock wave in postshock region, fluctuations increase several times, compared to the foreshock $\delta B / B=0.5-0.6$; 
- in deep magnetosheath region, fluctuations decrease to a value of $\delta B / B \sim 0.15-0.2$.

Even with almost complete absence of magnetic field fluctuations in the solar wind, there are high fluctuations in the magnetosheath region.

\section{RESULTS}

To study the probability density function of fluctuations of the magnetic field $B(t)$ we chose the offset time $\tau$, which is a multiple of discrete measurements $\tau_{\min }=0.0445 \mathrm{~s}$, and we analysed the statistical properties of the absolute value of the magnetic field variations $d B=B(t+\tau)-B(t)$ in the foreshock region, postshock region, and magnetosheath, on different time scales.

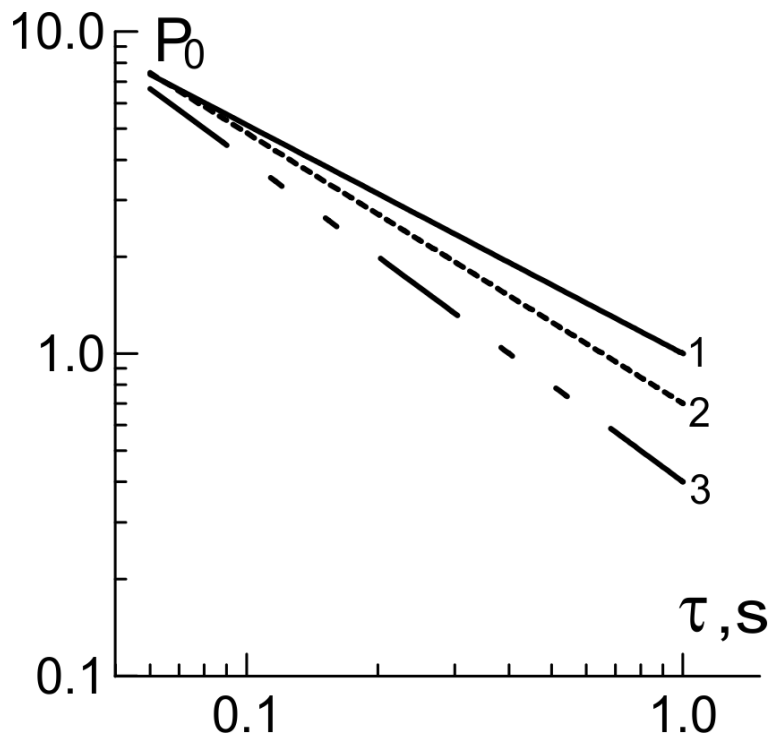

Fig. 2: Maximal value for magnetic field fluctuations PDF $P(0)$ in logarithmic scale for 3rd March 2004. Experimental data approximated with a line: $P \sim t^{-s}: 1$ - foreshock, 2 - magnetosheath, 3 - postshock. Values of $s$ presented in Table 1.

Good temporal resolution of the measurement allows us to observe the dependence of the maximum value of the PDF of magnetic field fluctuations $P_{\tau}(0)$ on a small scale (less than 1 second), which is very important because we can analyse on a scale smaller than the ion cyclotron frequency. Logarithmic scale plots for measurements on March 27, 2010 and March 20, 2006 are shown in Fig. 2, where the experimental points were approximated by a straight line $P_{\tau} \sim \tau^{-s}$. The values for the exponents of the events are presented in Table 1 .

From the obtained values, we can conclude that for small scales in the transition region of the Earth's magnetosphere, the distribution significantly deviates from the Gaussian distribution.

The largest deviations are observed for the postshock region. Furthermore, in foreshock region we have an almost Gaussian distribution for magnetic field fluctuations. The generalized diffusion coefficient $R$ is shown in Table 1. $R>0$ indicates the existence of superdiffusion.

The extended self-similarity structure functions of high orders are specified by the equation: $S_{q}(t)=$ $\left\langle|B(t+\tau)-B(t)|^{q}\right\rangle \sim \tau^{\zeta(q)}$, where $\langle\ldots\rangle$ - statistical averaging of experimental data over time.

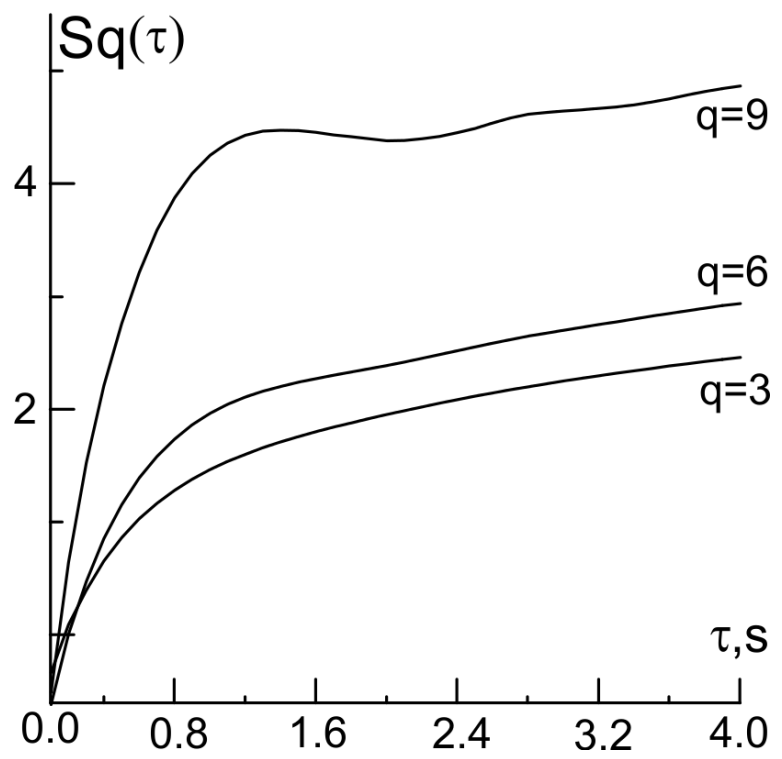

Fig. 3: Relation of structural function on time scale for a magnetosheath region $(03 / 03 / 2004)$.

Structural scaling function normalized to scaling for the third moment, $\zeta(q) / \zeta(3)$ can be obtained from the slope of a logarithmic scale. This will allow us to compare experimental data to the Kolmogorov model of turbulence (K41) for which $\zeta(3)=3 / 3=1$. In Fig. 3 power law $S_{q}(\tau) \sim \tau^{\zeta(q)}$ (i. e. self-similarity) is observed only in a limited range of time scales. This interval corresponds to the inertial range, which is considered a classic model of developed isotropic turbulence (K41, etc.) [5]. In the transition region of the magnetosphere this interval is observed on scales less than 1 second (this will allow us to compare the results obtained by different approaches - from the analysis of maximum height of magnetic field fluctuation probability density function and statistical average).

Calculated results for $\zeta(q) / \zeta(3)$ of different orders $q$ in the analysis of small-scale turbulence and comparison with Kolmogorov model for the events on 3rd March 2004 and 3rd April 2006 are shown in Fig. 4 and 5 . The most significant deviation from the Kolmogorov model is observed in the postshock region. Determined by the ESS analysis parameters $\beta$ and $\Delta$ and calculated values $R=0.3 \div 0.98$ are presented in Table 2.

The obtained values are in good agreement with the data presented in Table 2. 
Thus, using two independent techniques, we proved the existence of superdiffusion processes in the transition region of the Earth's magnetosphere.

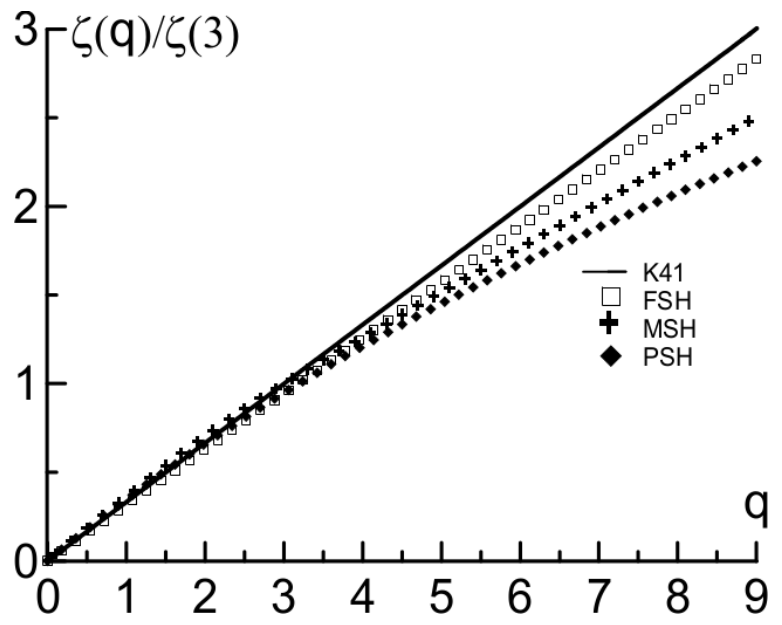

Fig. 4: Relation of exponential value of structural function of $q$-order to the 3-rd order structural function for 3rd March 2004. K41 — values calculated for Kolmogorov models; FSH — foreshock region, MSH - magnetosheath, PSH — postshock.

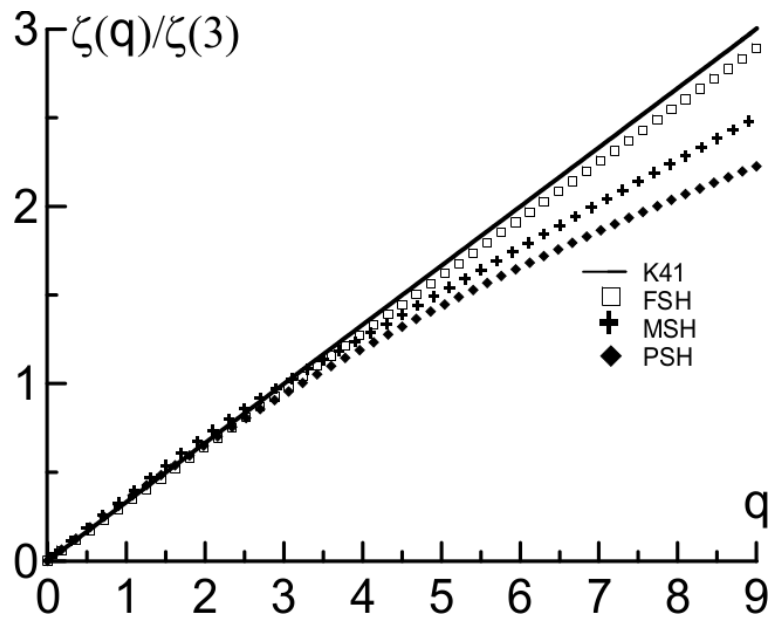

Fig. 5: Relation of exponential value of structural function of $q$-order to the 3-rd order structural function for 3rd April 2006. K41 — values calculated for Kolmogorov models; FSH — foreshock region, MSH - magnetosheath, PSH - postshock.

\section{CONCLUSIONS}

The relative variations of the magnetic field in magnetosheath exceed the values in the solar wind by factor of $2-5$. However, the converse proposition is incorrect - not all variations of parameters in the magnetosheath are generated by fluctuations of the solar wind or the interplanetary magnetic field.

The largest deviation from a Gaussian process is observed in the postshock region, and the distribu- tion closest to a Gaussian one is in the foreshock region.

The value of the generalized diffusion coefficient increases with time-scaling. Thus, the analysis of the evolution of the magnetic fluctuations PDF the exponent field dependence on the generalized diffusion coefficient of the scale $(R)$ in the transition regions of the Earth's magnetosphere: foreshock region, postshock and magnetosheath - is in the range of $0.32-0.92$. The highest value is observed for the postshock region. The analysis of the statistical properties of points determined the parameter $R$ to be in the range of $0.3-0.98$. Likewise, the highest values are observed for the postshock region in this case as well. It can be noted that different approaches to the analysis of turbulent processes give similar results and indicate the presence of superdiffusion processes in the transition region of the Earth's magnetosphere. This fact must be taken into account when constructing quantitative models of transport.

\section{ACKNOWLEDGEMENT}

The work is done in the frame of complex program of NAS of Ukraine on space research for 2012-1016, the grant Az.90 312 from the Volkswagen Foundation, and within the framework of the educational program No.2201250 "Education, Training of Students, PhD Students, Scientific and Sedagogical Staff Abroad" launched by the Ministry of Education and Science of Ukraine.

\section{REFERENCES}

[1] Benzi R., Ciliberto S., Tripiccione R. et al. 1993, Phys. Rev. E, 48, R29

[2] Chechkin A. V., Gorenflo R. \& Sokolov I. M. 2002, Phys. Rev. E, 66, 046129

[3] Consolini G., Kretzschmar M., Lui A. T. Y., Zimbardo G. \& Macek W. M. 2005, J. Geophys. Res., 110, A07202

[4] Dubrulle B. 1994, Phys. Rev. Lett., 73, 959

[5] Freak P. G. 1999, 'Turbulence: models and approaches', Perm National Technical University Press, Perm

[6] Frysh W. 1998, 'Turbulence: Kolmogorov heritage', Phasis, Moscow

[7] Hughes B. D. 1995, 'Random Walks and Random Environments, Volume 1: Random Walks', Oxford University Press, Oxford

[8] Klafter J., Shlesinger M. F. \& Zumofen G. 1996, Physics Today, 49, 33

[9] Kolmogorov A. N. 1941, Doklady Akademiia Nauk SSSR, 30, 301

[10] Kozak L. V. 2010, Space Science and Technology, 16, 1, 28

[11] Kozak L. V., Pilipenko V. A., Chugunova O. M. \& Kozak P. N. 2011, Cosmic Research, 49, 194

[12] Kraichnan R. H. 1959, J. Fluid Mechanics, 5, 497

[13] Kraichnan R. H. 1970, J. Fluid Mechanics, 41, 189 
Table 1: Generalized diffusion coefficient calculated from the evolution of magnetic field fluctuation PDF.

\begin{tabular}{llcc}
\hline Date & Region & $\mathrm{s}$ & $R=2 s-1$ \\
\hline $2009 / 03 / 23$ & Postshock & $0.78 \pm 0.085$ & 0.56 \\
& Magnetosheath & $0.69 \pm 0.092$ & 0.38 \\
\hline $2006 / 04 / 03$ & Foreshock & $0.66 \pm 0.076$ & 0.32 \\
& Postshock & $0.96 \pm 0.098$ & 0.92 \\
& Magnetosheath & $0.89 \pm 0.088$ & 0.78 \\
\hline $2009 / 05 / 01$ & Magnetosheath & $0.9 \pm 0.099$ & 0.81 \\
\hline $2004 / 03 / 03$ & Foreshock & $0.76 \pm 0.083$ & 0.52 \\
& Postshock & $0.96 \pm 0.087$ & 0.92 \\
& Magnetosheath & $0.66 \pm 0.054$ & 0.32 \\
\hline $2010 / 03 / 31$ & Magnetosheath & $0.68 \pm 0.069$ & 0.36 \\
\hline
\end{tabular}

Table 2: Generalized diffusion coefficient calculated from ESS analysis.

\begin{tabular}{lllcc}
\hline Date & Region & $\beta$ & $\Delta$ & $R=\Delta\left(\frac{1}{\beta}-1\right)$ \\
\hline $2004 / 03 / 03$ & Foreshock & $0.26 \pm 0.012$ & $0.2 \pm 0.01$ & 0.58 \\
& Postshock & $0.45 \pm 0.017$ & $0.8 \pm 0.011$ & 0.98 \\
& Magnetosheath & $0.66 \pm 0.009$ & $0.6 \pm 0.009$ & 0.3 \\
\hline $2006 / 04 / 03$ & Foreshock & $0.67 \pm 0.008$ & $0.69 \pm 0.007$ & 0.35 \\
& Postshock & $0.71 \pm 0.013$ & $2.2 \pm 0.01$ & 0.91 \\
& Magnetosheath & $0.45 \pm 0.01$ & $0.68 \pm 0.007$ & 0.82 \\
\hline $2009 / 03 / 23$ & Postshock & $0.5 \pm 0.012$ & $0.6 \pm 0.013$ & 0.6 \\
& Magnetosheath & $0.67 \pm 0.008$ & $0.69 \pm 0.007$ & 0.35 \\
\hline $2009 / 05 / 01$ & Magnetosheath & $0.5 \pm 0.011$ & $0.4 \pm 0.012$ & 0.81 \\
\hline $2010 / 03 / 31$ & Magnetosheath & $0.62 \pm 0.009$ & $0.51 \pm 0.008$ & 0.31 \\
\hline
\end{tabular}

[14] Landau L. D., Lifshitz E. M. \& Pitaevskii L.P. 1985, 'Electrodynamics of Continuous Media', ButterworthHeinemann, Oxford

[15] Metzler R. \& Klafter J. 2000, Phys. Rep., 339, 1

[16] Metzler R. \& Klafter J. 2001, Advances Chem. Phys., 116,223

[17] Oraevskij V. N., Konikov Yu. V. \& Khazanov G. V. 1985, 'Transport Processes in an Anisotropic Nearspace Plasma', Nauka, Moscow
[18] Richardson L. F. 1926, Proc. Roy. Soc. of London, 110, 709

[19] She Z.-S. \& Leveque E. 1994, Phys. Rev. Lett., 72, 336

[20] Shlesinger M. F., Zaslavsky G. M. \& Klafter J. 1993, Nature, 363, 31

[21] Sokolov I. M., Klafter J. \& Blumen A. 2002, Physics Today, 55, 48

[22] Treumann R. A. 1997, Geophys. Res. Lett., 24, 1727

[23] Iroshnikov P.S. 1963, AJ, 40, 742 